\title{
Oxidative Stress in Hypertension: Mechanisms and Therapeutic Opportunities
}

Authors

Affiliation

\section{R. Brito, G. Castillo, J. González, N. Valls, R. Rodrigo}

Laboratory of Oxidative Stress and Nephrotoxicity, Molecular and Clinical Pharmacology Program, Institute of Biomedical Sciences, Faculty of Medicine, University of Chile

\author{
Key words \\ - hypertension \\ - cardiovascular risk \\ management \\ - oxidative stress
}

\section{Abstract $\nabla$}

Hypertension is a highly prevalent disease worldwide. It is known for being one of the most important risk factors for developing cardiovascular disease, including acute myocardial infarction and stroke. Therefore, during the last decades there have been multiple efforts to fully understand the mechanisms underlying hypertension, and then develop effective therapeutic interventions to attenuate the morbidity and mortality associated with this condition. In this regard, oxidative stress has been proposed as a key mechanistic mediator of hypertension, which is an imbalance between oxidant species and the antioxidant defense systems. A large amount of evidence supports the role of vascular wall as a major source of reactive oxygen species. These include the activation of enzymes, such as

\section{Introduction}

first decision 10.12

accepted 27.02.2015

Bibliography

Dol http://dx.doi.org/

10.1055/s-0035-1548765

Published online:

April 28, 2015

Exp Clin Endocrinol Diabetes

2015; 123: 325-335

(c) J. A. Barth Verlag in Georg Thieme Verlag KG Stuttgart · New York ISSN 0947-7349

Correspondence

Dr. R. Rodrigo

University of Chile

Independencia 1027

Santiago

Chile 8380000

rrodrigo@med.uchile.cl
Hypertension is a highly prevalent pathological condition that is considered the most important risk factor for the occurrence of cardiovascular disease (Yusuf et al., 2004). During the last decades, oxidative stress has gained attention as one of the fundamental mechanisms responsible for the development of hypertension. Reactive oxygen species (ROS) have an important role in the homeostasis of the vascular wall, hence they could contribute to the mechanism of hypertension (Lassègue and Griendling, 2004; Paravicini and Touyz, 2006; Rodrigo et al., 2003). Thus, increased ROS production, and reduced nitric oxide (NO) and antioxidants bioavailability were demonstrated in experimental and human hypertension. Vascular superoxide is derived primarily from NADPH oxidase (NOX) when stimulated by hormones such as angiotensin II (AT-II), endothelin-1 (ET-1) and urotensin II (UT-II),
NADPH oxidase and xanthine oxidase, the uncoupling eNOS and mitochondrial dysfunction, having as a major product the superoxide anion. Among the stimuli that increase the production of oxidative species can be found the action of some vasoactive peptides, such as angiotensin II, endothelin-1 and urotensin II. The oxidative stress state generated leads to a decrease in the biodisponibility of nitric oxide and prostacyclin, key factors in maintaining the vascular tone. The knowledge of the mechanisms mentioned above has allowed generating some therapeutic strategies using antioxidants as antihypertensives with different results. Further studies are required to position antioxidants as key agents in the treatment of hypertension. The current review summarize evidence of the role of oxidative stress in hypertension, emphasizing in therapeutic targets that can be consider in antioxidant therapy.

among others. In addition, increased ROS production may be generated by mechanical forces, which increase with hypertension. ROS-induced vasoconstriction results from increased intracellular calcium concentration, thereby contributing to the pathogenesis of hypertension (Paravicini and Touyz, 2006). Vasomotor tone is dependent upon a delicate balance between vasoconstrictor and vasodilator forces resulting from the interaction of the components of the vascular wall and the blood, and both of them can be altered by oxidative stress. These findings have stimulated the interest on antihypertensive therapies targeted to decrease ROS generation and/or increase NO bioavailability. This review examines the available studies pointing to a role of oxidative stress in the mechanism of production of high blood pressure, as well as the use of antioxidants in the prevention or treatment of this disorder. 


\section{Mechanisms of Hypertension Involving Oxidative Stress \\ $\nabla$}

\section{Endothelial dysfunction}

Endothelial dysfunction has been implicated in the pathophysiology of different forms of cardiovascular disease, including hypertension. It may be defined as impairment characterized by a shift of the actions of the endothelium toward reduced vasodilation, a proinflammatory state, and prothrombotic setting. These events lead to a state of vascular inflammation, which may be mediated, partly, by ROS formed by activated mononuclear cells.

\section{Vascular oxidative stress and hypertension}

Oxidative stress constitutes a unifying mechanism of injury of many types of disease processes, it occurs when there is an imbalance between the generation of ROS and the antioxidant defense systems in the body. The ROS family comprises many molecules that have divergent effects on cellular function. Importantly, many of these actions are associated with pathological changes observed in cardiovascular disease. The effects of ROS are mediated through redox-sensitive regulation of multiple signaling molecules and second messengers (Hool and Corry, 2007; Kimura et al., 2005; Yoshioka et al., 2006). Several studies have demonstrated that essential hypertensive patients and various animal models of hypertension produce excessive amount of ROS (Lacy et al., 2000; Redon et al., 2003; Stojiljkovic et al., 2002; Tanito et al., 2004; Touyz, 2004), and have abnormal levels of antioxidant status (Briones and Touyz, 2010), thereby contributing to the accumulating evidence that increased vascular oxidative stress could be involved in the pathogenesis of essential hypertension (Bengtsson et al., 2003; Paravicini and Touyz, 2006; Rodrigo et al., 2003). Recently, it was demonstrated a strong association between blood pressure and some oxidative stress-related parameters (Rodrigo et al., 2007). Other studies show that mouse models with genetic deficient in ROS-generating enzymes have lower blood pressure compared with wildtype counterparts (Gavazzi et al., 2006; Landmesser et al., 2003). In addition, in cultured vascular smooth muscle cells (VSMC) and isolated arteries from hypertensive rats and humans, ROS production is enhanced, redox-dependent signaling is amplified, and antioxidant bioactivity is reduced (Touyz and Schiffrin, 2001). Classical antihypertensive agents such as $\beta$-adrenergic blockers, angiotensin converting enzyme (ACE) inhibitors, angiotensin receptor antagonists, and calcium channel blockers may be mediated, in part, by decreasing vascular oxidative stress (Ghiadoni et al., 2003; Yoshida et al., 2004).

Sources of ROS in vascular wall

A variety of enzymatic and non-enzymatic sources of ROS exist in blood vessels. The best characterized source of ROS is NOX. In addition to NOX, several other enzymes may contribute to ROS generation, including xanthine oxidase (XO), NO synthase and the mitochondrion.

NADPH oxidase: NOX is the primary biochemical source of ROS in the vasculature, particularly of superoxide. The kidney and vasculature are rich sources of NOX-derived ROS, which under pathological conditions play an important role in renal dysfunction and vascular damage (Feairheller et al., 2009; Touyz, 2004). This system catalyses the reduction of molecular oxygen by NADPH as electron donor, thus generating superoxide. NOX is up-regulated in hypertension by humoral and mechanical signals. AT-II is the most studied stimulus, but ET-1 and UT-II may also participate in activation of NADPH oxidase, thereby resulting in increased ROS. Likely the most well known function of NOX derived superoxide is inactivation of NO to form peroxynitrite, leading to impaired endothelium dependent vasodilation and uncoupling of endothelial NO synthase (eNOS) to produce additional superoxide (Landmesser et al., 2003; Zou et al., 2004). In the vasculature, NOX activation has been strongly associated with hypertension (Lassègue and Clempus, 2003).

Xanthine oxidase: $\mathrm{XO}$ is also an important source for oxygen free radical present in the vascular endothelium (Lassègue and Clempus, 2003; Viel et al., 2008). It catalyzes the last two steps of purine metabolism. During this process oxygen is reduced to superoxide. There is evidence suggesting involvement of this enzyme in hypertension. Spontaneously hypertensive rats demonstrate elevated levels of endothelial xanthine oxidase and increased ROS production, which is associated with increased arteriolar tone (Feairheller et al., 2009). In addition to effects on the vasculature, XO may play a role in end-organ damage in hypertension (Laakso et al., 2004).

Uncoupled endothelial NO synthase: The primary function of eNOS is NO production which regulates vasodilation. Nevertheless, L-arginine and tetrahydrobiopterin (BH4) - 2 essential cofactors for its action - deficiency or oxidation are associated with uncoupling of the L-arginine-NO pathway resulting in decreased formation of NO, and increased eNOS-mediated generation of superoxide. NOX is the initial source of ROS. Superoxide combines with NO, which is synthesized by eNOS, to form peroxynitrite (Kuzkaya et al., 2003). In turn, peroxynitrite oxidizes and destabilizes eNOS to produce more superoxide (Laursen et al., 2001; Zou et al., 2004). Superoxide also leads to BH4 oxidation (in fact, BH4 is highly sensitive to oxidation), which promotes eNOS uncoupling and ROS production.

Mitochondrial dysfunction: The mitochondrion is a major source and target of ROS. Part of the superoxide produced in the intermembrane space may be carried to the cytoplasm (Han et al., 2003). Ubiquinol or coenzyme $Q$ is a source of superoxide when partially reduced (semiquinone form) and an antioxidant when fully reduced (Eto et al., 1992). Complex I produces most of the superoxide generated by mammalian mitochondria in vitro. Complexes II and IV are not normally significant sites of ROS production. Mild uncoupling very effectively decreases the high superoxide production that occurs from complex I. A reduction in antioxidant enzymatic activity in patients with hypertension has been reported (Zhou et al., 2006).

\section{Role of the vascular wall components}

The endothelium senses mechanical and hormonal stimuli. In response, it releases agents that regulate vasomotor function. There is no doubt that endothelium plays a regulatory and protective role by generating vasorelaxing substances. Under some pathophysiological circumstances, endothelium derived vasoconstricting factors, such as ET-1, AT-II, UT-II, superoxide anions, vasoconstrictor prostaglandins and thromboxane A2, can be released and contribute to the paradoxical vasoconstrictor effects. VSMC are fit not only for short-term regulation of the blood vessel diameter and therefore of blood pressure, but also for long-term adaptation, via structural remodeling. ROS medi- 
ate many of these pathophysiological processes. The adventitia can contribute to hypertension by either reducing NO bioavailability or participating in vascular remodeling through ROS.

\section{Role of vascular hormones and factors}

Nitric oxide: NO is known to play an important role as a key paracrine regulator of vascular tone. Physiologically, NO inhibits leukocyte-endothelial cell adhesion, VSMC proliferation and migration, and platelet aggregation to maintain the health of the vascular endothelium. Therefore it has many beneficial effects. The decrease in bioavailability of NO in the vasculature reduces vasodilatory capacity and contributes to hypertension. The enzyme that catalyzes the formation of NO from oxygen and arginine is NO synthase (NOS), which in fact is a whole family of enzymes. eNOS is the predominant NOS isoform in the vessel wall. Receptor-mediated agonist stimulation leads to rapid enzyme activation. In addition, shear stress and allosteric modulators are also an important modulator of eNOS activity (Michel et al., 1997). Except the vasorelaxing and antiproliferative properties per se, NO plays an important role in antagonizing the effects of AT-II, endothelins and ROS. NO diffuses as a gas to the adjacent smooth muscle where it interacts with different receptor molecules such as the soluble guanylyl cyclase. It is accepted that the normal production of NO plays a crucial role in the maintenance of the physiologic conditions within the cardiovascular system. L-arginine, a substrate for eNOS, seems to be promising in preserving NO formation. However, L-arginine failed to prevent blood pressure increase and left ventricle remodeling due to chronic treatment with methyl ester of N-nitro-L-arginine (L-NAME), an inhibitor of eNOS (Simko et al., 2005). The ACE inhibitor captopril completely prevented NOdeficient hypertension, yet without improving NOS activity. NO also has an ACE down-regulation effect. Thiols protect NO from oxidation by scavenging oxygen-free radicals and by forming nitrosothiols, both effects prolonging NO half-life and duration of NO action (Sládková et al., 2007; Zhang and Hogg, 2005). Reduced NO levels can be attributed to elevated levels of ROS. Superoxide combines with NO to form peroxynitrite that oxidizes BH4 and destabilizes eNOS to produce more superoxide (Kuzkaya et al., 2003; Laursen et al., 2001; Zou et al., 2004), thus further enhancing the development of oxidative stress. The balance between NO and AT-II in the vasomotor centers seems to play important role in the regulation of the sympathetic tone.

Acetylcholine: In vascular vessels, acetylcholine (ACh) induces endothelium-dependent dilation via production of endothelial factors, mainly NO. NO then diffuses to underlying VSMC, where it induces vascular smooth muscle cell relaxation. The diminution in NO bioavailability will lead to significantly reduced AChmediated vasodilation (Bitar et al., 2005; Pechánová, 2007). The consequence of an overall increase in ROS is a reduce bioavailability of NO.

Renin-Angiotensin system: The rennin-angiotensin system (RAS) plays a key role in the development of cardiovascular disease. AT-II is a potent vasoactive peptide that can be formed in vascular beds rich in ACE. When AT-II production increases above normal levels, it induces vascular remodeling and endothelial dysfunction in association with increases in levels of blood pressure. As a potent activator of NOX, AT-II contributes to the production of ROS (Hitomi et al., 2007; Touyz, 2004). In rats and mice made hypertensive by AT-II infusion, expression of
NOX subunits, oxidase activity, and generation of ROS are all increased (Landmesser et al., 2002). AT-II not only increases NOX activity but also upregulates superoxide dismutase activity, possibly to compensate for the increased ROS. In situations where this compensatory effect is efficient, ROS levels may appear normal even in the face of prooxidant. However, when ROS production becomes overwhelming, compensatory mechanisms are inadequate and pathophysiological consequences ensue (Taniyama and Griendling, 2003). Captopril and enalapril prevented blood pressure rise in young spontaneously hypertensive rats inhibiting ACE. Captopril, probably due to the antioxidant role of its thiol group, had more effective hypotensive effect than enalapril (Pechánová, 2007). In contrast, NO not solely antagonizes the effects of AT-II on vascular tone, cell growth, and renal sodium excretion, but also down-regulates the synthesis of ACE and AT1 receptors (Bitar et al., 2005). On the other hand, ACE inhibition up-regulates eNOS expression. The ability of AT-II to induce endothelial dysfunction is also due to its ability to downregulate soluble guanylyl cyclase, thereby leading to impaired NO/cGMP signaling. Recently, it has been proposed that $\mathrm{Ca} 2+$ / calmodulin-dependent protein kinase II is an important molecule linking AT-II, ROS and cardiovascular pathological conditions (Wen et al., 2012).

Endothelin-1: Endothelins are potent vasoconstrictor isopeptides produced in different vascular tissues, including vascular endothelium. ET-1 is the main endothelin generated by the endothelium and the most important in the cardiovascular system. When ET- 1 is administered in large concentrations, it behaves as a potent vasoconstrictor capable of exerting an array of physiological effects, including the potential to alter arterial pressure. ET- 1 mediates its effects through 2 receptors, ETA and ETB. ETA mediates contractions via activation of NOX, XO, lipoxygenase, uncoupled NOS, and mitochondrial respiratory chain enzymes. The ETB induces relaxation on endothelial cells (Gomez-Alamillo et al., 2003). Many factors that normally stimulate ET-1 synthesis, (e.g., thrombin, AT-II) also cause the release of vasodilators such as prostacyclin (PGI2) and/or NO, which oppose the vasoconstricting action of ET-1. It was reported that essential hypertension is characterized by increased ET-1 vasoconstrictor tone, an effect that seems to be dependent on decreased endothelial ETB-mediated NO production attributable to the impaired NO bioavailability.

Urotensin-II: UT-II is a potent vasoactive peptide (Djordjevic et al., 2005), indeed the most potent vasoconstrictor identified. It acts trough activation of NOX. The role of UT-II in disease is not well elucidated. The constrictor response to UT-II appears to be variable and highly dependent on the vascular bed examined. Vasoconstriction is not its only effect, because UT receptors have been found in other organs (Jegou et al., 2006; Matsushita et al., 2001). UT-II has also been shown to act as a potent vasodilator in some isolated vessels (Stirrat et al., 2001).

Norepinephrine: VSMC is innervated primarily by the sympathetic nervous system through adrenergic receptors. 3 types of adrenoceptors are present within VSMC: $\alpha 1, \alpha 2$ and $\beta 2$. Norepinephrine stimulates VSMC proliferation. In addition, overexpression of inducible nitric oxide synthase (iNOS) increases blood pressure via central activation of the sympathetic nervous system, which is mediated by an increase in oxidative stress (Kimura et al., 2005). 
Prostaglandins: PGI2, another endothelium-dependent vasodilator, relaxes the VSMC. PGI2 is released in higher amount in response to ligand binding such as thrombin, arachidonic acid, histamine, or serotonin. The enzymes prostaglandin $\mathrm{H} 2$ synthase uses arachidonic acid as a substrate, forming prostaglandin $\mathrm{H} 2$. Prostaglandin $\mathrm{H} 2$ is converted to vasoactive molecules, such as PGI2. The isoform prostaglandin H2 synthase-2 may mediate vascular dysfunction in conditions characterized by oxidative stress. Thus, peroxynitrite inhibits the enzymatic activity of prostacyclin synthase, thereby causing impairment in the PGI2-mediated vasodilation.

Homocysteine: This molecule may play an important role in the pathogenesis of essential hypertension (Rodrigo et al., 2003a). Elevated homocysteinemia diminishes the vasodilation by NO, increases oxidative stress, stimulates the proliferation of VSMC, and alters the elastic properties of the vascular wall. Thus, homocysteine contributes to elevate the blood pressure (Rodrigo et al., 2003b). It is also known that elevated homocysteine levels could lead to oxidant injury of the endothelium (Rodrigo et al., 2003a). The correction of elevated homocysteinemia by administration of vitamins B12 and B6 plus folic acid, could be a useful adjuvant therapy of hypertension (Harrison and Gongora, 2009; Rodrigo et al., 2003a). However, further controlled randomized trials are necessary to establish the efficacy of these therapeutic agents.

A hypothesis for the role of vascular oxidative stress in hypertension is depicted in $\bullet$ Fig. 1.

This review has discussed the importance of ROS in the vasculature and its relation to hypertension, but it is important to emphasize the evidence that hypertensive stimuli, such as high salt and AT-II, promote the production of ROS not only in the vasculature, but also in kidney and the central nervous system (CNS) and that each of these sites contributes either to hypertension or to the untoward sequels of this disease (Harrison and Gongora, 2009).

\section{Role of oxidative stress in kidney}

Evidence proposes that ROS play a key role in the pathophysiological processes of several renal diseases; these diseases are considered to be cause and consequence of hypertension. Regarding glomerular alterations, ROS mediates lipoprotein glomerulopathy and other inflammatory glomerular lesions (Rodrigo and Rivera, 2002). A recent study demonstrates that NOX activation and production of ROS through lipid raft clustering is an important molecular mechanism triggering oxidative injury of podocytes induced by homocysteine. This may represent an early event initiating glomerulosclerosis during hyperhomocysteinemia (Zhang et al., 2010). Concerning ROS mediated tubulointerstitial injury, one of the mechanisms is the exposure of tubular cells to LDL which may result in tubulointerstitial damage due to ROS production mediated by NOX (Piccoli et al., 2011). AT-II also plays a pivotal role in the progression of tubulointersitial injury but also in obstructive nephropathy (Grande et al., 2010; Klahr, 2001). It activates NOX and, subsequently, generates superoxide that leads to hypertrophy of renal tubular cells (Sachse and Wolf, 2007).

There is evidence suggesting that a high-fat diet induces renal inflammation and aggravation of blood pressure in spontaneously hypertensive rats, via ROS (Chung et al., 2010). It is also known that the metabolic syndrome is a risk factor for chronic kidney disease (CKD) at least in part independent of diabetes and hypertension per se, probably mediated by ROS. Moreover, the onset and maintenance of renal damage may worsen metabolic syndrome features like hypertension, leading to potential vicious cycles (Guarnieri et al., 2010).

There are several oxidative stress-mediated mechanisms involved in endothelial dysfunction in CKD (Malyszko, 2010). ROS are elevated in CKD and related to endothelium-dependent vascular reactivity and systolic blood pressure (Costa-Hong et al., 2009). High ROS and increased level of the endogenous asymmetric dimethylarginine (ADMA) was reported to be a novel risk factor for endothelial dysfunction (Zoccali et al., 2001). More-

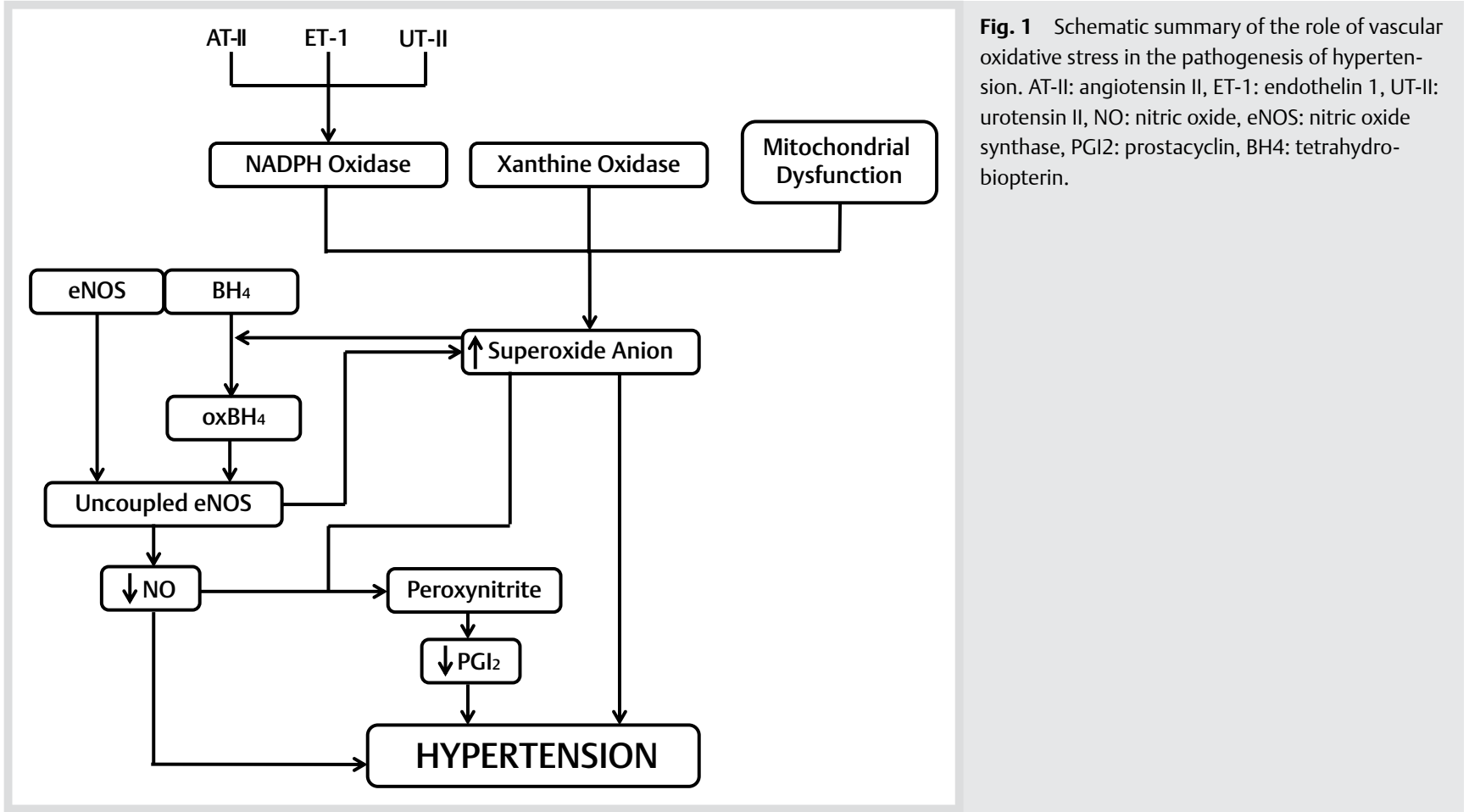


over, high levels of ADMA were reported in CKD and were associated with higher intima-media thickness and cardiovascular events (Nanayakkara et al., 2005). In renovascular hypertension, oxidative stress in the ischemic kidney plays a major role in the maintenance of hypertension in 2 kidney-one clip rats (Campos et al., 2011).

\section{Role of oxidative stress in central nervous system}

Just like the kidney and the vasculature itself, the sympathetic nervous system (SNS), regulated in the CNS, plays an important role in the pathogenesis of hypertension (Grassi, 2009). Recent studies strongly suggest that central sympathetic outflow is increased in hypertension (Guyenet, 2006). There is also evidence that increased ROS generation in the brainstem contributes to the neural mechanisms of hypertension in hypertensive rats (Kishi et al., 2004).

The rostral ventrolateral medulla (RVLM) is the major vasomotor center and is essential for the maintenance of basal vasomotor tone (Hirooka et al., 2010; Sved et al., 2003). There are findings that strongly indicate that ROS in the RVLM is increased in stroke-prone spontaneously hypertensive rats and thereby contributes to the neural mechanisms of hypertension through activation of the SNS (Hirooka et al., 2010). The paraventricular nucleus of the hypothalamus is most likely also involved in the ROS mediated neural mechanism of hypertension (OliveiraSales et al., 2009). There is evidence that other regions of the brain are also involved in ROS mediated hypertension. These investigations suggest that increased intracellular superoxide production in the subfornical organ is critical in the development of AT-II-induced hypertension (Zimmerman et al., 2004).

\section{Antioxidants in Hypertension}

This section refers to the antihypertensive role of endogenous and exogenous antioxidants that have demonstrated their ability to alter the blood vessels function and to participate in the main redox reactions involved in the pathophysiology of hypertension.

\section{Vitamin C}

Vitamin C is a potent water-soluble antioxidant. On the vascular wall behaves as enzyme modulator exerting up-regulation on eNOS and down regulation of NOX (Ulker et al., 2003). Most studies have demonstrated an inverse relationship between plasma ascorbate levels and blood pressure in both normotensive and hypertensive populations (Rodrigo et al., 2007a). It has been shown that treatment with antioxidants improves the vascular function and reduces the blood pressure in animal models (Nishikawa et al., 2003; Reckelhoff et al., 1998) and in human hypertension (Atarashi et al., 1997; Chen et al., 2001). Vitamin C may have favorable effects on vascular dilation, possibly through its antioxidant effects on NO (Duffy et al., 2001; Jackson et al., 1998; Vita et al., 1998).

Nevertheless, there are several small and short-term clinical trials in which the effect of vitamin $C$ supplements on blood pressure have yielded inconsistent findings (Block et al., 2001; Duffy et al., 1999; Fotherby et al., 2000; Galley et al., 1997; Ghosh et al., 1994; Mullan et al., 2002). The lack of antihypertensive efficacy observed in studies using supplementation with vitamin $C$ alone could be due to the decreased bioavailability of NO under conditions of oxidative stress. It was shown that these effects are mediated in part by the ability of vitamin $\mathrm{C}$ to protect $\mathrm{BH} 4$ from oxidation and thereby increase the enzymatic activity of eNOS (Steinbrenner and Sies, 2009). In addition, this uncertain clinical beneficial effect of vitamin $C$ in vivo as an antihypertensive agent could be due to the lack of consideration of their pharmacokinetic properties. It was experimentally determined that the antihypertensive effect of vitamin $C$ is expected to occur at a concentration by $10 \mathrm{mM}$ (Jackson et al., 1998), a plasma level unreachable in humans through oral administration, but that would be required to compete efficiently with the reaction of NO with superoxide. The renal ascorbic acid threshold occurs at vitamin C dose between 60 and $100 \mathrm{mg}$ daily. Plasma is completely saturated at doses of $400 \mathrm{mg}$ daily and higher, producing a steady-state plasma concentration of approximately $80 \mu \mathrm{M}$ (Padayatty et al., 2003). Thus, the antihypertensive effect may only be active in plasma following vitamin $\mathrm{C}$ infusion at high doses.

\section{Vitamin E}

This major lipid-soluble antioxidant has received considerable attention for their antioxidant potential. Epidemiological data support a role of high dietary vitamin E intake and a reduced incidence of cardiovascular disease (Malyszko, 2010). Increasing evidence indicates that vitamin E can act as a biological modifier independently of its antioxidant activity. Experimental evidence available shows that vitamin E is capable of dose-dependently regulating mitochondrial generation of superoxide and hydrogen peroxide.

However, intervention trials have not been convincing, with a number of studies demonstrating no beneficial effect of vitamin E on cardiovascular disease outcomes (Lee et al., 2005; Lonn et al., 2005; Rapola et al., 1997; Ward et al., 2007). Moreover, a study using supplementation with vitamin E, either as $\alpha$-tocopherol or mixed tocopherols, showed a significant increase in blood pressure, pulse pressure and heart rate in individuals with type 2 diabetes (Ward et al., 2007). It should be noted that it is unlikely to achieve sufficiently high concentrations in the vascular microenvironment to interfere effectively with all components of oxidative stress (Münzel and Keaney, 2001).

\section{Association of Vitamins C and E}

Ascorbic acid may reduce the $\alpha$-tocopheroxyl radical and may be required for beneficial vascular effects of $\alpha$-tocopherol (Heller et al., 2006). In fact, the effect of $\alpha$-tocopherol seems to be dependent on tissue saturation with vitamin $C$, and both vitamins may act synergistically to provide optimal conditions for endothelial NO formation (Heller et al., 2004). Thus, the association of vitamins $\mathrm{C}$ and $\mathrm{E}$ is expected to have an antihypertensive effect probably because this combined therapy provides a reinforcement of their individual properties through a complementary effect (Bilodeau and Hubel, 2003).

Despite the biological effects of both vitamin C and E, long-term clinical trials have failed to consistently support their antihypertensive effects in patients at high cardiovascular risk. Some short-term trials have shown that supplemental antioxidant vitamin intake lowers blood pressure (Fotherby et al., 2000; Galley et al., 1997; Mullan et al., 2002; Plantinga et al., 2007) but the majority of clinical long-term trials did not find any antihypertensive effects of antioxidant vitamins. However, most of these studies lack rigorous exclusion criteria in the selection of subjects to avoid the influence of confounders (Rodrigo et al., 
2007b). It deserves special mention that regarding cohorts included in large trials, most subjects had irreversible cardiovascular disease.

\section{Selenium}

Selenium is an essential trace element and an integral part of many proteins with catalytic and structural functions. It exerts an antioxidant function mainly in the form of selenocysteine residues, an integral constituent of ROS-detoxifying selenoenzymes, such as glutathione peroxidase (GSH-Px), thioredoxin reductases (TR) and selenoprotein $\mathrm{P}$ (Miller et al., 2001). Maintenance of full GSH-Px and TR activity by adequate dietary selenium supply has been proposed to be useful for the prevention of several cardiovascular disorders (Steinbrenner and Sies, 2009). In addition, selenium is capable of preventing the union of nuclear factor kappa B (NF-kB) to its nuclear response elements in DNA (Faure et al., 2004). NF-kB has a key role in inflammation and production of ROS. The inhibition of NF-KB is presumed to be the result of the binding of the selenium to the essential thiols of this transcription factor (Kim and Stadtman, 1997).

Its antioxidant properties have been documented in several trials (Brigelius-Flohé et al., 2003; Campbell et al., 2007; Faure, 2003; Faure et al., 2004; Ito and Fujita, 1996; Takizawa et al., 2007; Zhou et al., 2007). Selenium at low doses can provide significant protection of the human coronary artery endothelium against damage by oxidative stress (Miller et al., 2001). In an animal model, dietary supplementation with selenium was associated with lower levels of cardiac oxidative damage and increased antioxidant expression, as well as a reduction in disease severity and mortality in spontaneously hypertensive rats (Lymbury et al., 2010). A reduced selenium concentration in hypertensive pregnancies has been associated with a diminution of GSH-Px activity (Mistry et al., 2008). Thus it is reasonable to say that deficiency of selenium might be an underestimated risk factor for the development of high blood pressure (Nawrot et al., 2007).

\section{Allopurinol}

$\mathrm{XO}$ is an important source of ROS in the vascular endothelium (Kuzkaya et al., 2003). It catalyzes the last two steps of purine metabolism, producing uric acid. XO activity is associated with an increasing arteriolar tone and therefore, hypertension (DeLano et al., 2006; Suzuki et al., 1998). XO inhibitors such as allopurinol have shown marked improvements in endothelial function in various cohorts at risk of cardiovascular events. Treatment with allopurinol result in reduction of blood pressure in adolescents (Feig et al., 2008), spontaneously hypertensive rats (Mazzali et al., 2001) and patients with CKD (Goicoechea et al., 2010). Nevertheless, most of the evidence so far comes from smaller mechanistic studies, and the few large randomized controlled trials have not shown significant mortality benefit using these agents (George and Struthers, 2009).

\section{Polyphenols}

Polyphenols are the most abundant antioxidant in diet. They can act as ROS scavengers, iron chelators, enzyme modulators (Pietta et al., 1998; Rodrigo and Bosco, 2006), and possibly enhancing the production of NO (Duarte et al., 2004; Zenebe et al., 2003). In humans, after the consumption of polyphenols, circulating NO concentration increases (Pechánová et al., 2006b). Polyphenols also increase glutathione, and inhibit ROS-producing enzymes such as NOX and XO. These pathways lead to improved endothe- lial function (Rodrigo et al., 2012). However, some studies have shown increased blood pressure by association of polyphenols with vitamin C (Ward et al., 2005).

\section{$\mathrm{N}$-acetylcysteine}

The antioxidant $\mathrm{N}$-acetylcysteine (NAC), a sulfhydryl group donor, improves renal dysfunction and decreases arterial pressure and renal injury in salt-sensitive hypertension (Tian et al., 2006). The inhibition of oxidative stress in hypertensive states probably contributes to the therapeutic effects of $\mathrm{N}$-acetylcysteine, an effect likely mediated by an NO-dependent mechanism (Pechánová et al., 2006a). This protective mechanism is exerted by prevention of $\mathrm{BH} 4$ oxidation by the increased superoxide (Zembowicz et al., 1993). In addition, this molecule can protect against oxidative damage inhibiting lipid peroxidation and scavenging ROS (De la Fuente and Victor, 2001; Penugonda et al., 2005).

\section{Diet}

There is sufficient evidence to suggest that dietary approaches may help to prevent and control high blood pressure. There are dietary approaches regarding the prevention and management of hypertension: i.e., moderate use of sodium, alcohol, an increased potassium intake, plant fibers, calcium, and foods like salmon, nuts, wine, among others (Appel et al., 1997). In a Mediterranean population with an elevated fat consumption, a high fruit and vegetable intake is inversely associated with blood pressure levels (Alonso et al., 2004). Short-term studies indicate that specialized diets may prevent or ameliorate mild hypertension, most notable are the Dietary Approaches to Stop Hypertension (DASH) diet, which is high in fruits, vegetables, and low-fat dairy products (Savica et al., 2010). It has been reported that a low sodium DASH diet is effective in reducing blood pressure in hypertensive patients, particularly in those taking antihypertensive medications (Nowson et al., 2009). In addition, DASH diet had significant beneficial effects on cardiovascular risk (Azadbakht et al., 2011; Chen et al., 2010; Levitan et al., 2009). In overweight or obese persons with above-normal blood pressure, the addition of exercise and weight loss to the DASH diet resulted in even larger blood pressure reductions, greater improvements in vascular and autonomic function, and reduced left ventricular mass (Blumenthal et al., 2010; Smith et al., 2010).

\section{Clinical Trials using Antioxidants as Antihypertensive Therapy

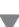

Recent advances in understanding the complexity of redox signaling in the vascular system points to a central role of oxidative stress in the pathogenesis of vascular dysfunction. This is how hypertension is associated with impaired endothelium-dependent vasodilation with inactivation of endothelium-derived nitric oxide by oxygen free radicals. In this regard, it has arisen a growing interest concerning the therapeutic possibilities to target ROS in the management of essential hypertension.

In support of this view, epidemiological studies suggest that individuals with higher antioxidant intake have reduced cardiovascular risk. In fact, population-based observational studies have shown an inverse association between diverse plasma antioxidant concentrations, obtained by dietary intake, with blood pressure (Moran et al., 1993; Nawrot et al., 2007), providing justification for trials evaluating antioxidant supplementation as 
adjunct anti-hypertensive therapy favoring blood pressure reduction.

Antihypertensive effects of vitamin C were hypothesized as early as 1946 (Hoitink, 1946), and it has been proven that vitamin $C$ enhances endothelial function through effects on nitric oxide production (Jackson et al., 1998). Most studies have demonstrated an inverse relation between vitamin C plasma levels and blood pressure, in normotensive and hypertensive populations (Houston, 2005; Laakso et al., 2004). However, evidence for blood pressure-lowering effects of vitamin $C$ in clinical trials is still inconsistent. Nevertheless, laboratory (Ettarh et al., 2002; Yoshioka et al., 1985) and human studies (Feldman et al., 1992; Koh, 1984) have established biological plausibility for a clinical use of antioxidants concerning hypertension.

Taddei et al. made one of the first trials in 1998 (Taddei et al., 1998), where patients with essential hypertension received intra-arterial infusion of vitamin $C$, and showing that in essential hypertensive patients vitamin $C$ significantly increased the vasodilation effect of the muscarinic agonist, acetylcholine, indicating that antioxidant vitamin $\mathrm{C}$ improves endotheliumdependent vasodilation in hypertensive patients. As ratifying evidence, On et al. in 2002 (On et al., 2002) conducted a study that achieved similar results on endothelium dysfunction, using vitamin $C$ as an adjunctive therapy to amlodipine.

Despite the evidence points to the use of vitamin $C$ as an adjunct in the treatment on essential hypertension, there is still lack of long-term studies that support its use. Up to date there are few trials that have used chronic supplementation. In a small randomized, double-blind controlled trial (Hajjar et al., 2002), patients were followed for 8 months and were randomized to receive 500,1000 and $2000 \mathrm{mg}$ of vitamin C once daily. Results of this study showed a significant diminution of both, mean systolic blood pressure and diastolic blood pressure, with no differences between the increasing doses of vitamin C. Additionally, these effects were only seen during the first month of supplementation, suggesting only a short term benefit. Besides this, other trial aimed to study the effects of ascorbic acid on ambulatory blood pressure in elderly patients, showing that chronic supplementation of vitamin C ( $600 \mathrm{mg} /$ daily $)$ markedly reduced systolic blood pressure and pulse pressure in ambulatory patients (Sato et al., 2006). Furthermore, this was accompanied by decreases of oxidative stress biomarkers such as levels of 8-isoprostane and malondialdehyde.

The strongest evidence of the possible role of vitamin $C$ on hypertension treatment was handed by a recent a meta-analysis that included 29 trials, concluding that in short-term trials, vitamin C supplementation reduces systolic and diastolic blood pressure. But it also highlights that long-term trials on the effects of vitamin C on blood pressure and clinical events are still needed to elucidate its true benefit (Juraschek et al., 2012).

Because supplementation made only with vitamin $\mathrm{C}$ has achieved inconsistent clinical outcomes, the scientific rational approach has led to the suggestion that the combined intake of antioxidants could achieve better clinical results. To prove this concept, a small randomized double-blind placebo-controlled trial was made including 38 subjects, 21 hypertensive and 17 normotensive (Galley et al., 1997). Groups were assigned to receive in a crossover design placebo or a combination of antioxidants consisting of zinc, ascorbic acid, alpha-tocopherol and beta-carotene daily for 8 weeks. Although it was a short-term following, this combined therapy of antioxidants showed that systolic blood pressure fell significantly on hypertensive sub- jects while been on the antioxidant phase compared with placebo. Additional evidence was given by another study aimed to evaluate the effect of short-term combined treatment with antioxidants vitamin C and E (Plantinga et al., 2007): 30 essential hypertensive patients were assigned randomly either to vitamin C plus vitamin E or placebo for 8 weeks. Results showed that parameters of flow-mediated dilation of the brachial artery and central pulse wave velocity were significantly improved after antioxidant supplementation, concluding that treatment with vitamins $C$ and $E$ has beneficial effects on endothelium-dependent vasodilation in untreated essential hypertensive patients.

Following the same consideration, recently a randomized double-blind placebo-controlled clinical trial was conducted to test the hypothesis that oral administration of vitamin $\mathrm{C}$ and $\mathrm{E}$ together causes decrease in blood pressure in patients with mild-to-moderate essential hypertension, 110 men with recent diagnosis of grade 1 essential hypertension were randomly assigned to receive either vitamin C (1g) plus Vitamin E (400UI) daily or placebo for 8 weeks. The results of this study, showed for the first time a specific association between oxidative-stress related parameters and blood pressure. Following administration of vitamins $C$ plus $\mathrm{E}$, patients with essential hypertension had significantly lower systolic, diastolic and mean arterial blood pressure (Rodrigo et al., 2008).

According to the theoretical possibility of the role of antioxidants, further trials have been performed using different compounds with antioxidant activity. This is how Barrios et al. in 2002 conducted a patient crossover study with the aim to investigate the potential effect of NAC added to the ACE inhibitors antihypertensive action. A significant decrease in systolic and diastolic blood pressure was achieved with the combination of ACE inhibitors and NAC compared to ACE inhibitors-only period (Barrios et al., 2002).

A more recent study tried the use of melatonin to evaluate its effectiveness as an adjunct for a combined treatment adding melatonin to standard anti-hypertensive drugs (Zaslavskaia et al., 2010). This study showed that combined therapy had better outcomes than standard therapy alone on essential hypertensive patients.

Although there is objective compelling evidence supporting the use of antioxidants in the management of hypertensive patients, there are also several studies that have not shown beneficial effects. As an example: Vitamin E (Palumbo et al., 2000), Coenzyme Q10 (Young et al., 2012), NAC (Schneider et al., 2005) and vitamin C (Kim et al., 2002) have failed to obtain beneficial effects on clinical settings.

A summary of the antioxidant approaches as clinical interventions on essential hypertension is presented on $\odot$ Table 1.

\section{Conclusions and Perspectives}

$\nabla$

There is considerable evidence supporting the view that oxidative stress is involved in the pathophysiology of hypertension. ROS are mediators of the major physiological vasoconstrictors, increasing intracellular calcium concentration. In addition, superoxide reduces the bioavailability of NO and enhances superoxide production via uncoupled eNOS, further enhancing oxidative stress, a major factor of hypertension.

Antioxidant therapy can curtail the development of hypertension in animal models, but remains controversial in humans. Possible confounding factors in patients include co-existing 
Table 1 Clinical trials accounting for strategies using antioxidants in essential hypertension.

\author{
Details of Study \\ Intrabrachial vitamin C $(2.4 \mathrm{mg} / 100 \mathrm{~mL}$ forearm tissue per \\ minute). Randomized, Placebo-controlled trial \\ Intra-arterial infusion of vitamin C at $24 \mathrm{mg} / \mathrm{min}$ for $10 \mathrm{~min}$ \\ Randomized trial \\ Oral administration of 500,1000 or $2000 \mathrm{mg}$ of vitamin C \\ once daily. Randomized, double-blind, placebo-controlled \\ trial \\ Chronic supplementation of $600 \mathrm{mg} /$ daily of vitamin C. \\ Randomized, placebo-controlled trial \\ Included 29 trials of vitamin C supplementation. Meta- \\ analysis \\ Oral supplementation: \\ $1 \mathrm{~g}$ Vitamin C \\ 400 UI Vitamin E \\ or \\ Placebo for 8 weeks. Randomized, double-blind, placebo- \\ controlled, crossover study \\ Oral supplementation: \\ $1 \mathrm{~g}$ Vitamin C \\ 400 UI Vitamin E \\ Placebo for 8 weeks. Randomized double-blind placebo- \\ controlled trial \\ ACE inhibitors plus \\ NAC (600 mg t.i.d.) \\ Or \\ ACE inhibitors only. Randomized, controlled trial, cross- \\ over study \\ Intra-arterial administration: \\ NAC $(48 \mathrm{~g} / \mathrm{min})$ \\ or \\ Vitamin C (18 mg/min). Cross-over randomized study \\ Vitamin C supplement daily. Either $50 \mathrm{mg}$ or $500 \mathrm{mg}$, for 5 \\ years. Randomized double-blind controlled trial
}

\section{Results}

In hypertensive patients but not in control subjects,

Vitamin C increased the impaired vasodilation to acetylcholine

Forearm blood flow response to acetylcholine was significantly enhanced with intra-arterial infusion of vitamin $C$ in hypertensive group before antihypertensive treatment

Significant diminution of mean systolic blood pressure and diastolic blood pressure, with no differences between the increasing doses of vitamin C

Reduced systolic blood pressure and pulse pressure in ambulatory elderly patients, but not in adult group

In short-term trials, vitamin C supplementation reduces systolic and diastolic blood pressure

Treatment with vitamins $C$ and $E$ has beneficial effects on endothelium-dependent vasodilation in untreated essential hypertensive patients

Specific association between oxidative-stress related parameters and blood pressure

Patients with essential hypertension had significantly lower systolic, diastolic and mean arterial blood pressure

Significant decrease in systolic and diastolic blood pressure with the combination of ACE inhibitors and NAC compared to ACE inhibitors-only

The intra-arterial administration of NAC had no effect on endothelium-dependent vasodilation Intra-arterial Vitamin C improved endothelium-dependent vasodilation

Neither systolic nor diastolic blood pressure was significantly related with the serum vitamin $C$ concentration
Barrios et al., 2010

Schneider et al., 2005

\section{Reference}

Koh, 1984

Taddei et al., 1998

Hajjar et al., 2002

Sato et al., 2006

Juraschek et al., 2012

Plantinga et al., 2007

Rodrigo et al., 2008

Kim et al., 2002 pathologies and treatments, lack of selection of treatments according to ROS levels, among others. However, the dietary intake of antioxidants and polyphenols could have an effect in the primary prevention or reduction of hypertension. Despite existing molecular basis and in-vitro evidence supports the use of diverse antioxidants, clinical evidence continues to be controversial. It is necessary to collect efforts in performing basic/ clinical trials that augment the current, which could eventually help to elucidate the role of antioxidants as novel therapy for essential hypertension. Also available data lead us to think that antioxidants may not play the same role in different stages of disease, suggesting that supplementation could be only beneficial during the phase of endothelial dysfunction, which precedes an established vascular damage. In this setting antioxidants would be more likely to have a role on early stages of hypertension with the potential to reverse or counteract deleterious effects of ROS. In contrast, it should not be expected an antihypertensive effect in patients having an advanced state of cardiovascular disease, in which chronic damaging effects of oxidative stress may be unreachable for antioxidant approach.

\section{Conflict of interest: None.}

\section{References}

1 Alonso A, de la Fuente C, Martín-Arnau AM et al. Fruit and vegetable consumption is inversely associated with blood pressure in a Mediterranean population with a high vegetable-fat intake: the Seguimiento Universidad de Navarra (SUN) Study. Br J Nutr 2004; 92: 311-319

2 Appel LJ, Moore TJ, Obarzanek E et al. A clinical trial of the effects of dietary patterns on blood pressure. DASH Collaborative Research Group. N Engl J Med 1997; 336: 1117-1124

3 Atarashi K, Ishiyama A, Takagi M et al. Vitamin E ameliorates the renal injury of Dahl Salt-sensitive rats. Am J Hypertens 1997; 10: 116-119

4 Azadbakht L, Fard NR, Karimi M et al. Effects of the Dietary Approaches to Stop Hypertension (DASH) eating plan on cardiovascular risks among type 2 diabetic patients: A Randomized Cross-Over Clinical Trial. Diabetes Care 2011; 34: 55-57

5 Barrios V, Calderón A, Navarro-Cid J et al. $\mathrm{N}$-acetylcysteine potentiates the antihypertensive effect of ACE inhibitors in hypertensive patients. Blood Press 2002; 11: 235-239

6 Bengtsson SH, Gulluyan LM, Dusting GJ et al. Novel isoforms of NADPH oxidase in vascular physiology and pathophysiology. Clin Exp Pharmacol Physiol 2003; 30: 849-854

7 Bilodeau JF, Hubel CA. Current concepts in the use of antioxidants for the treatment of preeclampsia. J Obstet Gynaecol Can 2003; 25: $742-750$

8 Bitar MS, Wahid S, Mustafa S et al. Nitric oxide dynamics and endothelial dysfunction in type II model of genetic diabetes. Eur J Pharmacol 2005; 511: 53-64

9 Block G, Mangels AR, Norkus EP et al. Ascorbic acid status and subsequent diastolic and systolic blood pressure. Hypertension 2001; 37: 261-267 
10 Blumenthal JA, Babyak MA, Hinderliter A et al. Effects of the DASH diet alone and in combination with exercise and weight loss on blood pressure and cardiovascular biomarkers in men and women with high blood pressure: the ENCORE study. Arch Intern Med 2010; 170: 126-135

11 Brigelius-Flohé R, Banning A, Schnurr K. Selenium-dependent enzymes in endothelial cell function. Antioxid Redox Signal 2003; 5: 205-215

12 Briones AM, Touyz RM. Oxidative stress and hypertension: current concepts. Curr Hypertens Rep 2010; 12: 135-142

13 Campbell L, Howie F, Arthur JR et al. Selenium and sulforaphane modify the expression of selenoenzymes in the human endothelial cell line EAhy926 and protect cells from oxidative damage. Nutrition 2007; 23: 138-144

14 Campos RR, Oliveira-Sales EB, Nish EM et al. The role of oxidative stress in renovascular hypertension Special Series: Stress and Hypertension. Clin Exp Pharmacol Physiol 2011; 38: 144-152

15 Chen ST, Maruthur NM, Appel LJ. The effect of dietary patterns on estimated coronary heart disease risk: results from the Dietary Approaches to Stop Hypertension (DASH) trial. Circ Cardiovasc Qual Outcomes 2010; 3: 484-489

16 Chen X, Touyz RM, Park JB et al. Antioxidant effects of vitamins $C$ and $E$ are associated with altered activation of vascular NADPH oxidase and superoxide dismutase in stroke-prone SHR. Hypertension 2001; 38: $606-611$

17 Chung S, Park CW, Shin SJ et al. Tempol or candesartan prevents highfat diet-induced hypertension and renal damage in spontaneously hypertensive rats. Nephrol Dial Transplant 2010; 25: 389-399

18 Costa-Hong V, Bortolotto LA, Jorgetti $V$ et al. Oxidative stress and endothelial dysfunction in chronic kidney disease. Arq Bras Cardiol 2009; 92: 381-386

19 De la Fuente M, Victor VM. Ascorbic acid and N-acetylcysteine improve in vitro the function of lymphocytes from mice with endotoxin-induced oxidative stress. Free Radic Res 2001; 35: 73-84

20 DeLano FA, Parks DA, Ruedi JM et al. Microvascular display of xanthine oxidase and NADPH oxidase in the spontaneously hypertensive rat. Microcirculation 2006; 13: 551-566

21 Djordjevic T, BelAiba RS, Bonello $S$ et al. Human urotensin II is a novel activator of NADPH oxidase in human pulmonary artery smooth muscle cells. Arterioscler Thromb Vasc Biol 2005; 25: 519-525

22 Duarte J, Andriambeloson E, Diebolt $M$ et al. Wine polyphenols stimulate superoxide anion production to promote calcium signaling and endothelial-dependent vasodilatation. Physiol Res 2004; 53: 595602

23 Duffy SJ, Gokce N, Holbrook M et al. Treatment of hypertension with ascorbic acid. Lancet 1999; 354: 2048-2049

24 Duffy SJ, Gokce N, Holbrook M et al. Effect of ascorbic acid treatment on conduit vessel endothelial dysfunction in patients with hypertension. Am J Physiol Heart Circ Physiol 2001; 280: 528-534

25 Ettarh RR, Odigie IP, Adigun SA. Vitamin C lowers blood pressure and alters vascular responsiveness in salt-induced hypertension. Can J Physiol Pharmacol 2002; 80: 1199-1202

26 Eto Y, Kang D, Hasegawa E et al. Succinate-dependent lipid peroxidation and its prevention by reduced ubiquinone in beef heart submitochondrial particles. Arch Biochem Biophys 1992; 295: 101-106

27 Faure P. Protective effects of antioxidant micronutrients (vitamin E, zinc and selenium) in type 2 diabetes mellitus. Clin Chem Lab Med 2003; 41: 995-998

28 Faure P, Ramon O, Favier A et al. Selenium supplementation decreases nuclear factor-kappa $B$ activity in peripheral blood mononuclear cells from type 2 diabetic patients. Eur J Clin Invest 2004; 34: 475-481

29 Feairheller DL, Brown MD, Park JY et al. Exercise training, NADPH oxidase p22phox gene polymorphisms, and hypertension. Med Sci Sports Exerc 2009; 41: 1421-1428

30 Feig DI, Soletsky B, Johnson RJ. Effect of allopurinol on blood pressure of adolescents with newly diagnosed essential hypertension: a randomized trial. JAMA 2008; 300: 924-932

31 Feldman EB, Gold S, Greene $J$ et al. Ascorbic acid supplements and blood pressure:a four-week pilot study. Ann N Y Acad Sci 1992; 669: 342-344

32 Fotherby $M D$, Williams JC, Forster LA et al. Effect of vitamin C on ambulatory blood pressure and plasma lipids in older persons. J Hypertens 2000; 18: 411-415

33 Galley HF, Thornton J, Howdle PD et al. Combination oral antioxidant supplementation reduces blood pressure. Clin Sci (Lond) 1997; 92: 361-365

34 Gavazzi G, Banfi B, Deffert C et al. Decreased blood pressure in NOX1deficient mice. FEBS Lett 2006; 580: 497-504
35 George J, Struthers A. The role of urate and xanthine oxidase in vascular oxidative stress: future directions. Ther Clin Risk Manag 2009; 5: 799-803

36 Ghiadoni L, Magagna A, Versari D et al. Different effect of antihypertensive drugs on conduit artery endothelial function. Hypertension 2003; 41: 1281-1286

37 Ghosh SK, Ekpo EB, Shah IU et al. A double-blind, placebo-controlled parallel trial of vitamin $C$ treatment in elderly patients with hypertension. Gerontology 1994; 40: 268-272

38 Goicoechea M, Vinuesa SG, Verdalles $U$ et al. Effect of Allopurinol in Chronic Kidney Disease Progression and Cardiovascular Risk. Clin J Am Soc Nephrol 2010; 5: 1388-1393

39 Gomez-Alamillo C, Juncos LA, Cases A et al. Interactions between vasoconstrictors and vasodilators in regulating hemodynamics of distinct vascular beds. Hypertension 2003; 42: 831-836

40 Grande MT, Pérez-Barriocanal F, López-Novoa JM. Role of inflammation in túbulo-interstitial damage associated to obstructive nephropathy. J Inflamm (Lond) 2010; 7: 19-32

41 Grassi G. Assessment of sympathetic cardiovascular drive in human hypertension: Achievements and perspectives. Hypertension 2009; 54: 690-697

42 Guarnieri G, Zanetti M, Vinci P et al. Metabolic syndrome and chronic kidney disease. J Ren Nutr 2010; 20: 19-23

43 Guyenet PG. The sympathetic control of blood pressure. Nat Rev Neurosci 2006; 7: 335-346

44 Hajjar IM, George V, Sasse EA et al. A randomized, double-blind, controlled trial of vitamin $C$ in the management of hypertension and lipids. Am J Ther 2002; 9: 289-293

45 Han D, Antunes F, Canali $R$ et al. Voltage-dependent anion channels control the release of the superoxide anion from mitochondria to cytosol. J Biol Chem 2003; 278: 5557-5563

46 Harrison DG, Gongora MC. Oxidative stress and hypertension. Med Clin North Am 2009; 93: 621-635

47 Heller R, Werner-Felmayer G, Werner ER. Alpha-tocopherol and endothelial nitric oxide synthesis. Ann N Y Acad Sci 2004; 1031: 74-85

48 Heller $R$, Werner-Felmayer $G$, Werner ER. Antioxidants and endothelial nitric oxide synthesis. Eur J Clin Pharmacol 2006; 62: 21-28

49 Hirooka $Y$, Sagara $Y$, Kishi $T$ et al. Oxidative stress and central cardiovascular regulation. Pathogenesis of hypertension and therapeutic aspects. Circ J 2010; 74: 827-835

50 Hitomi $H$, Kiyomoto $H$, Nishiyama A. Angiotensin II and oxidative stress. Curr Opin Cardiol 2007; 22: 311-315

51 AWJH Hoitink. Research on the influence of vitamin C administration on the human organism, in particular in connection with the working capacity. Verh Nederlands Inst Praevent 1946; 4: 62-63

52 Hool LC, Corry B. Redox control of calcium channels: from mechanisms to therapeutic opportunities. Antioxid Redox Signal 2007; 9: 409-435

53 Houston MC. Nutraceuticals, vitamins, antioxidants, and minerals in the prevention and treatment of hypertension. Prog Cardiovasc Dis 2005; 47: 396-449

54 Ito $Y$, Fujita T. Trace elements and blood pressure regulation. Nippon Rinsho 1996; 54: 106-110

55 Jackson TS, Xu A, Vita JA et al. Ascorbate prevents the interaction of superoxide and nitric oxide only at very high physiological concentrations. Circ Res 1998; 83: 916-922

56 Jegou S, Cartier D, Dubessy C et al. Localization of the urotensin II receptor in the rat central nervous system. J Comp Neurol 2006; 495: 21-36

57 Juraschek SP, Guallar E, Appel LJ et al. Effects of vitamin C supplementation on blood pressure: a meta-analysis of randomized controlled trials. Am J Clin Nutr 2012; 95: 1079-1088

$58 \mathrm{Kim}$ MK, Sasaki S, Sasazuki S et al. Lack of long-term effect of vitamin C supplementation on blood pressure. Hypertension 2002; 40: 797803

59 Kim IY, Stadtman TC. Inhibition of NF-kappaB DNA binding and nitric oxide induction in human $T$ cells and lung adenocarcinoma cells by selenite treatment. Proc Natl Acad Sci 1997; 94: 12904-12907

60 Kimura S, Zhang GX, Nishiyama A et al. Mitochondria-derived reactive oxygen species and vascular MAP kinases: comparison of angiotensin II and diazoxide. Hypertension 2005; 45: 438-444

61 Kishi T, Hirooka Y, Kimura $Y$ et al. Increased reactive oxygen species in rostral ventrolateral medulla contribute to neural mechanisms of hypertension in stroke-prone spontaneously hypertensive rats. Circulation 2004; 109: 2357-2362

62 Klahr S. Urinary tract obstruction. Semin Nephrol 2001; 21: 133-145 
63 Koh ET. Effect of vitamin C on blood parameters of hypertensive subjects. J Okla State Med Assoc 1984; 77: 177-182

64 Kuzkaya N, Weissmann N, Harrison DG et al. Interactions of peroxynitrite, tetrahydrobiopterin, ascorbic acid, and thiols: implications for uncoupling endothelial nitric-oxide synthase. J Biol Chem 2003; 278: 22546-22554

65 Laakso JT, Teräväinen TL, Martelin E et al. Renal xanthine oxidoreductase activity during development of hypertension in spontaneously hypertensive rats. J Hypertens 2004; 22: 1333-1340

66 Lacy F, Kailasam MT, O'Connor DT et al. Plasma hydrogen peroxide production in human essential hypertension: role of heredity, gender, and ethnicity. Hypertension 2000; 36: 878-884

67 Landmesser U, Cai H, Dikalov S et al. Role of p47(phox) in vascular oxidative stress and hypertension caused by angiotensin II. Hypertension 2002; 40: 511-515

68 Landmesser U, Dikalov S, Price SR et al. Oxidation of tetrahydrobiopterin leads to uncoupling of endothelial cell nitric oxide synthase in hypertension. J Clin Invest 2003; 111: 1201-1209

69 Lassègue B, Clempus RE. Vascular NAD(P)H oxidases: specific features, expression, and regulation. Am J Physiol Regul Integr Comp Physiol 2003; 285: 277-297

70 Lassègue B, Griendling $K$. Reactive Oxygen Species in Hypertension, An Update. Am J Hypertens 2004; 17: 852-860

71 Laursen JB, Somers M, Kurz S et al. Endothelial regulation of vasomotion in apoE-deficient mice: implications for interactions between peroxynitrite and tetrahydrobiopterin. Circulation 2001; 103: 12821288

72 Lee IM, Cook NR, Gaziano JM et al. Vitamin E in the primary prevention of cardiovascular disease and cancer: the Women's Health Study: a randomized controlled trial. JAMA 2005; 294: 56-65

73 Levitan EB, Wolk A, Mittleman MA. Relation of consistency with the dietary approaches to stop hypertension diet and incidence of heart failure in men aged 45 to 79 years. Am J Cardiol 2009; 104: 14161420

74 Lonn E, Bosch J, Yusuf S et al. HOPE and HOPE-TOO Trial Investigators. Effects of long-term vitamin $\mathrm{E}$ supplementation on cardiovascular events and cancer: a randomized controlled trial. JAMA 2005; 293 : 1338-1347

75 Lymbury RS, Marino MJ, Perkins AV. Effect of dietary selenium on the progression of heart failure in the ageing spontaneously hypertensive rat. Mol Nutr Food Res 2010; 54: 1436-1444

76 Malyszko J. Mechanism of endothelial dysfunction in chronic kidney disease. Clin Chim Acta 2010; 411: 1412-1420

77 Matsushita M, Shichiri M, Imai T et al. Co-expression of urotensin II and its receptor (GPR14) in human cardiovascular and renal tissues. J Hypertens 2001; 19: 2185-2190

78 Mazzali M, Hughes J, Kim Y et al. Elevated Uric Acid Increases Blood Pressure in the Rat by a Novel Crystal-Independent Mechanism. Hypertension 2001; 38: 1101-1106

79 Michel JB, Feron O, Sase K et al. Caveolin versus calmodulin. Counterbalancing allosteric modulators of endothelial nitric oxide synthase. J Biol Chem 1997; 272: 25907-25912

80 Miller S, Walker SW, Arthur JR et al. Selenite protects human endothelial cells from oxidative damage and induces thioredoxin reductase. Clin Sci (Lond) 2001; 100: 543-550

81 Mistry HD, Wilson V, Ramsay MM et al. Reduced selenium concentrations and glutathione peroxidase activity in preeclamptic pregnancies. Hypertension 2008; 52: 881-888

82 Moran JP, Cohen L, Greene JM et al. Plasma ascorbic acid concentrations relate inversely to blood pressure in human subjects. Am J Clin Nutr 1993; 57: 213-217

83 Mullan BA, Young IS, Fee $\mathrm{H}$ et al. Ascorbic acid reduces blood pressure and arterial stiffness in type 2 diabetes. Hypertension 2002; 40: 804-809

84 Münzel T, Keaney JF Jr. Are ACE inhibitors a "magic bullet" against oxidative stress? Circulation 2001; 104: 1571-1574

85 Nanayakkara PW, Teerlink T, Stehouwer CD et al. Plasma asymmetric dimethylarginine (ADMA) concentration is independently associated with carotid intima-media thickness and plasma soluble vascular cell adhesión molecule-1 (sVCAM-1) concentration in patients with mild-to-moderate renal failure. Kidney Int 2005; 68: 2230-2236

86 Nawrot TS, Staessen JA, Roels HA et al. Blood pressure and blood selenium: a cross-sectional and longitudinal population study. Eur Heart J 2007; 28: 628-633

87 Nishikawa Y, Tatsumi K, Matsuura T et al. Effects of vitamin C on high blood pressure induced by salt in spontaneously hypertensive rats. J Nutr Sci Vitaminol (Tokyo) 2003; 49: 301-309
88 Nowson CA, Wattanapenpaiboon N, Pachett A. Low-sodium Dietary Approaches to Stop Hypertension-type diet including lean red meat lowers blood pressure in postmenopausal women. Nutr Res 2009; 29: $8-18$

89 Oliveira-Sales EB, Nishi EE, Carillo BA et al. Oxidative stress in the sympathetic promotor neurons contributes to sympathetic activation in renovascular hypertension. Am J Hypertens 2009; 22: 484-492

90 On YK, Kim CH, Sohn DW et al. Improvement of endothelial function by amlodipine and vitamin $C$ in essential hypertension. Korean J Intern Med 2002; 17: 131-137

91 Padayatty SJ, Katz A, Wang $Y$ et al. Vitamin C as an antioxidant: evaluation of its role in disease prevention. J Am Coll Nutr 2003; 22: 18-35

92 Palumbo G, Avanzini F, Alli $C$ et al. Effects of vitamin E on clinic and ambulatory blood pressure in treated hypertensive patients. Collaborative Group of the Primary Prevention Project (PPP) - Hypertension study. Am J Hypertens 2000; 13: 564-567

93 Paravicini TM, Touyz RM. Redox signalling in hypertension. Cardiovasc Res 2006; 71: 247-258

94 Pechánová O,Zicha J, Kojsová S et al. Effect of chronic N-acetylcysteine treatment on the development of spontaneous hypertension. Clin Sci (Lond) 2006; 110: 235-242

95 Pechánová O, Rezzani R, Babál P et al. Beneficial effects of Provinols: cardiovascular system and kidney. Physiol Res 2006; 55: 17-30

96 Pechánová 0 . Contribution of captopril thiol group to the prevention of spontaneous hypertension. Physiol Res 2007; 56: 41-48

97 Penugonda S, Mare S, Goldstein G et al. Effects of N-acetylcysteine amide (NACA), a novel thiol antioxidant against glutamate-induced cytotoxicity in neuronal cell line PC12. Brain Res 2005; 1056: 132138

98 Plantinga Y, Ghiadoni L, Magagna A et al. Suplementation with vitamins $C$ and $E$ improves arterial stiffness and endothelial function in essential hypertensive patients. Am J Hypertens 2007; 20: 392-397

99 Piccoli C, Quarato G, D'Aprile A et al. Native LDL-induced oxidative stress in human proximal 1 tubular cells: multiple players involved. J Cell Mol Med 2011; 15: 375-395

100 Pietta P, Simonetti P, Gardana C et al. Relationship between rate and extent of catechin absorption and plasma antioxidant status. Biochem Mol Biol Int 1998; 46: 895-903

101 Rapola JM, Virtamo J, Ripatti S et al. Randomised trial of a-tocopherol and b-carotene supplements on incidence of major coronary events in men with previous myocardial infarction. Lancet 1997; 349: $1715-1720$

102 ReckelhoffJF, Kanji V, Racusen LC et al. Vitamin E ameliorates enhanced renal lipid peroxidation and accumulation of $\mathrm{F} 2$-isoprostanes in aging kidneys. Am J Physiol 1998; 274: 767-774

103 Redon J, Oliva MR, Tormos C et al. Antioxidant activities and oxidative stress byproducts in human hypertension. Hypertension 2003; 41: 1096-1101

104 Rodrigo R, Rivera G. Renal damage mediated by oxidative stress: a hypothesis of protective effects of red wine. Free Radic Biol Med 2002; 33: 409-422

105 Rodrigo R, Passalacqua $W$, Araya $J$ et al. Implications of oxidative stress and homocysteine in the pathophysiology of essential hypertension. J Cardiovasc Pharmacol 2003; 42: 453-461

106 Rodrigo R, Passalacqua W, Araya J et al. Homocysteine and essentia hypertension. J Clin Pharmacol 2003; 43: 1299-1306

107 Rodrigo $R$, Bosco $C$. Oxidative stress and protective effects of polyphenols: comparative studies in human and rodent kidney. A review. Comp Biochem Physiol C Toxicol Pharmacol 2006; 142: 317-327

108 Rodrigo $R$, Prat $H$, Passalacqua W et al. Relationship between oxidative stress and essential hypertension. Hypertens Res 2007; 30: 11591167

109 Rodrigo R, Guichard C, Charles R. Clinical pharmacology and therapeutic use of antioxidant vitamins. Fundam Clin Pharmacol 2007; 21: $111-127$

110 Rodrigo $R$, Prat H, Passalacqua $W$ et al. Decrease in oxidative stress through supplementation of vitamins $C$ and $E$ is associated with a reduction in blood pressure in patients with essential hypertension. Clin Sci (Lond) 2008; 114: 625-634

111 Rodrigo R, Gil D, Miranda-Merchak A et al. Antihypertensive role of polyphenols. Adv Clin Chem 2012; 58: 225-254

112 Sachse A, Wolf $G$. Angiotensin II-induced reactive oxygen species and the kidney. J Am Soc Nephrol 2007; 18: 2439-2446

113 Sato K, Dohi Y, Kojima $M$ et al. Effects of ascorbic acid on ambulatory blood pressure in elderly patients with refractory hypertension. Arzneimittelforschung 2006; 56: 535-540 
114 Savica $V$, Bellinghieri G, Kopple JD. The effect of nutrition on blood pressure. Annu Rev Nutr 2010; 30: 365-401

115 Schneider MP, Delles C, Schmidt BM et al. Superoxide scavenging effects of $\mathrm{N}$-acetylcysteine and vitamin $\mathrm{C}$ in subjects with essential hypertension. Am J Hypertens 2005; 18: 1111-1117

116 Simko F, Luptak I, Matuskova J et al. L-arginine fails to protect against myocardial remodelling in L-NAME-induced hypertension. Eur J Clin Invest 2005; 35: 362-368

117 Sládková M, Kojsová S, Jendeková L et al. Chronic and acute effects of different antihypertensive drugs on femoral artery relaxation of L-NAME hypertensive rats. Physiol Res 2007; 56: 85-91

118 Smith PJ, Blumenthal JA, Babyak MA et al. Effects of the dietary approaches to stop hypertension diet, exercise, and caloric restriction on neurocognition in overweight adults with high blood pressure. Hypertension 2010; 55: 1331-1338

119 Steinbrenner $H$, Sies $H$. Protection against reactive oxygen species by selenoproteins. Biochim Biophys Acta 2009; 1790: 1478-1485

120 Stirrat A, Gallagher M, Douglas SA et al. Potent vasodilator responses to human urotensin-II in human pulmonary and abdominal resistance arteries. Am J Physiol Heart Circ Physiol 2001; 280: 925-928

121 Stojiljkovic MP, Lopes HF, Zhang $D$ et al. Increasing plasma fatty acids elevates F2-isoprostanes in humans: implications for the cardiovascular risk factor cluster. J Hypertens 2002; 20: 1215-1221

122 Suzuki H, DeLano FA, Parks DA et al. Xanthine oxidase activity associated with arterial blood pressure in spontaneously hypertensive rats. Proc Natl Acad Sci USA 1998; 95: 4754-4759

123 Sved $A F$, Ito $S$, Sved JC. Brainstem mechanisms of hypertension: Role of the rostral ventrolateral medulla. Curr Hypertens Rep 2003; 5: 262-268

124 Taddei S, Virdis A, Ghiadoni L et al. Vitamin C improves endotheliumdependent vasodilation by restoring nitric oxide activity in essential hypertension. Circulation 1998; 97: 2222-2229

125 Takizawa M, Komori K, Tampo $Y$ et al. Paraquat-induced oxidative stress and dysfunction of cellular redox systems including antioxidative defense enzymes glutathione peroxidase and thioredoxin reductase. Toxicol In Vitro 2007; 21: 355-363

126 Tanito M, Nakamura H, Kwon YW et al. Enhanced oxidative stress and impaired thioredoxin expression in spontaneously hypertensive rats. Antioxid Redox Signal 2004; 6: 89-97

127 Taniyama Y, Griendling K. Reactive Oxygen Species in the Vasculature: Molecular and Cellular Mechanisms. Hypertension 2003; 42: 10751081

128 Tian $N$, Rose RA, Jordan $S$ et al. N-Acetylcysteine improves renal dysfunction, ameliorates kidney damage and decreases blood pressure in salt-sensitive hypertension. J Hypertens 2006; 24: 2263-2270

129 Touyz RM, Schiffrin EL. Increased generation of superoxide by angiotensin II in smooth muscle cells from resistance arteries of hypertensive patients: role of phospholipase D-dependent $\mathrm{NAD}(\mathrm{P}) \mathrm{H}$ oxidase-sensitive pathways. J Hypertens 2001; 19: 1245-1254

130 Touyz RM. Reactive oxygen species, vascular oxidative stress, and redox signaling in hypertension: what is the clinical significance? Hypertension 2004; 44: 248-252

131 Ulker S, McKeown PP, Bayraktutan U. Vitamins reverse endothelial dysfunction through regulation of eNOS and NAD(P)H oxidase activities. Hypertension 2003; 41: 534-539

132 Viel EC, Benkirane K, Javeshghani D et al. Xanthine oxidase and mitochondria contribute to vascular superoxide anion generation in DOCA-salt hypertensive rats. Am J Physiol Heart Circ Physiol 2008; 295: 281-288

133 Vita JA, Frei B, Holbrook $M$ et al. L-2-Oxothiazolidine-4-carboxylic acid reverses endothelial dysfunction in patients with coronary artery disease. J Clin Invest 1998; 101: 1408-1414
134 Ward NC, Hodgson JM, Croft KD et al. The combination of vitamin C and grape-seed polyphenols increases blood pressure: a randomized, double-blind, placebo-controlled trial. J Hypertens 2005; 23: 427434

135 Ward NC, Wu JH, Clarke MW et al. The effect of vitamin E on blood pressure in individuals with type 2 diabetes: a randomized, doubleblind, placebo-controlled trial. J Hypertens 2007; 25: 227-234

136 Wen H, Gwathmey JK, Xie LH. Oxidative stress-mediated effects of angiotensin II in the cardiovascular system. World J Hypertens 2012; 2: $34-44$

137 Yoshida J, Yamamoto K, Mano T et al. AT1 receptor blocker added to ACE inhibitor provides benefits at advanced stage of hypertensive diastolic heart failure. Hypertension 2004; 43: 686-691

138 Yoshioka M, Aoyama K, Matsushita T. Effects of ascorbic acid on blood pressure and ascorbic acid metabolism in spontaneously hypertensive rats (SH rats). Int J Vitam Nutr Res 1985; 55: 301-307

139 Yoshioka J, Schreiter ER, Lee RT. Role of thioredoxin in cell growth through interactions with signaling molecules. Antioxid Redox Signal 2006; 8: 2143-2145

140 Young JM, Florkowski CM, Molyneux SL et al. A randomized, doubleblind, placebo-controlled crossover study of coenzyme Q10 therapy in hypertensive patients with the metabolic syndrome. Am J Hypertens 2012; 25: 261-270

141 Yusuf S, Hawken S, Ounpuu S et al. Effect of potentially modifiable risk factors associated with myocardial infarction in 52 countries (the INTERHEART Study): case control study. Lancet 2004; 364: 937-952

142 Zaslavskaia RM, Shcherban' EA, Lilitsa GV et al. Melatonin in the combined treatment of cardiovascular diseases. Klin Med (Mosk) 2010; 88: $26-30$

143 Zembowicz A, Hatchett RJ, Radziszewski Wet al. Inhibition of endothelial nitric oxide synthase by ebselen. Prevention by thiols suggests the inactivation by ebselen of a critical thiol essential for the catalytic activity of nitric oxide synthase. J Pharmacol Exp Ther 1993; 267: 1112-1118

144 Zenebe $W$, Pechánová $O$, Andriantsitohaina $R$. Red wine polyphenols induce vasorelaxation by increased nitric oxide bioactivity. Physiol Res 2003; 52: 425-432

145 Zhang $Y$, Hogg N. S-Nitrosothiols: cellular formation and transport Free Radic Biol Med 2005; 38: 831-838

146 Zhang C, Hu JJ, Xia $M$ et al. Redox signaling via lipid raft clustering in homocysteine-induced injury of podocytes. Biochim Biophys Acta 2010; 1803: 482-491

147 Zhou L, Xiang W, Potts J et al. Reduction in extracellular superoxide dismutase activity in African-American patients with hypertension. Free Radic Biol Med 2006; 41: 1384-1391

148 Zhou X, Ji WJ, Zhu $Y$ et al. Enhancement of endogenous defenses against ROS by supra-nutritional level of selenium is more safe and effective than antioxidant supplementation in reducing hypertensive target organ damage. Med Hypotheses 2007; 68: 952-956

149 Zimmerman MC, Lazartigues E, Sharma RV et al. Hypertension caused by angiotensin II infusion involves increased superoxide production in the central nervous system. Circ Res 2004; 95: 210-216

150 Zoccali C, Bode-Böger S, Mallamaci F et al. Plasma concentration of asymmetrical dimethylarginine and mortality in patients with endstage renal disease: aprospective study. Lancet 2001; 358: 21132117

151 Zou MH, Cohen RA, Ullrich V. Peroxynitrite and vascular endothelial dysfunction in diabetes mellitus. Endothelium 2004; 11: 89-97 